\title{
Mensuração da atividade física e tempo sedentário por meio de acelerômetros: cenário atual, perspectivas e demandas futuras
}

Inácio Crochemore Mohnsam da Silva 1,2, Jeffer Eidi Sasaki ${ }^{3}$, Priscila Bezerra Gonçalves,

Os acelerômetros têm sido cada vez mais utilizados para mensurar a atividade física (AF) e o tempo sedentário (TS) de forma objetiva ${ }^{1}$, possibilitando estabelecer relações dose-resposta mais precisas com desfechos de saúde ${ }^{2}$. Apesar dos importantes avanços quanti e qualitativos da acelerometria, muitos desafios ainda persistem e serão discutidos ao longo deste editorial. O atual momento é propício para destacar pontos controversos, refletir sobre as tomadas de decisões metodológicas e suscitar diálogos e colaborações entre pesquisadores da área no Brasil.

Muitas das decisões metodológicas a serem tomadas no início de um estudo são complexas e merecem debate entre a comunidade científica, pois influenciam diretamente as estimativas finais de $\mathrm{AF}_{\mathrm{e}} \mathrm{TS}^{3}$. A primeira é a definição da marca e modelo do aparelho que pretende-se utilizar. Os principais aparelhos (ActiGraph e GeneActiv) disponibilizam atualmente os dados brutos, alta tecnologia e capacidade de registro, possibilitando maior liberdade analítica. Embora os aparelhos da ActiGraph sejam atualmente os mais utilizados entre os pesquisadores, a escolha pode ser baseada na preconização de maior comparabilidade, custo desses equipamentos, softwares e processo de importação. Em relação ao local de uso, a utilização dos acelerômetros no quadril tem sido a mais adotada até hoje, potencializando a comparabilidade entre estudos e apresentando maior disponibilidade de pontos de corte para classificar intensidades das AFs e o TS. Por outro lado, a utilização no punho tem apresentado maior aceitabilidade dos participantes e vem sendo priorizado, principalmente, em estudos de larga escala ${ }^{1}$.

A definição do número de dias de utilização dos acelerômetros também merece ser debatida. Para avaliar a AF e o TS habitual têm sido recomendado a utilização do aparelho durante sete dias consecutivos, permitindo capturar os dias de semana e finais de semana, os quais potencialmente apresentam diferentes padrões e tempos de utilização. Porém, quando essa recomendação não é atingida, seja pelo protocolo de pesquisa ou pela conduta dos participantes, umas das possibilidades é a utilização da média de tempo de AFs diárias. Não obstante, especificidades relacionadas ao grupo populacional, ao número de acelerômetros, recursos humanos, materiais e financeiros, bem como a necessidade de avaliar momentos diários específicos (por exemplo, aulas de Educação Física) podem influenciar estas decisões.

2 Grupo de Estudo e Pesquisa em Acelerometria (GEPEA), Programa de Pós-Graduação em Epidemiologia, Universidade Federal de Pelotas

3 Núcleo de Estudos em Atividade Física \& Saúde, Programa de Pós-Graduação em Educação Física, Universidade Federal do Triângulo Mineiro

4 Grupo de Pesquisa em Atividade Física e Qualidade de Vida (GPAQ), Pontifícia

Universidade Católica do Paraná

5 Programa de Pós-Graduação em Educação Física, Universidade Federal do Paraná 
Quanto ao critério para definir um dia como válido, estudos sugerem o uso mínimo de oito a dez horas por dia. O desafio, porém, está na variabilidade do tempo de utilização diária entre diferentes indivíduos e, consequentemente, nas estimativas de AF e TS. Nestes casos, pode-se utilizar o percentual de AF e TS do total de uso como forma de equiparar as estimativas entre pessoas com diferentes tempos de uso diário (10h vs. 20h). Estudos que identifiquem o impacto desses critérios são importantes na tomada de decisão. Outra possibilidade são estratégias de uso do equipamento 24 horas por dia, que anteriormente não eram possíveis em virtude da impossibilidade de diferenciação entre o período de sono, não uso e TS, bem como da falta de resistência do equipamento à água. Além disso, aspectos como a maior aceitabilidade da utilização no punho têm aumentado efetivamente o tempo de utilização do aparelho.

O processo de análise dos dados dos acelerômetros é tão importante quanto a padronização no processo de coleta. Erros analíticos comprometem a qualidade das informações geradas. As estimativas finais são diretamente relacionadas aos critérios de redução dos dados, os quais precisam ser discutidos entre os pesquisadores. Por exemplo, os pontos de corte precisam ser oriundos de estudos de calibração semelhantes ao grupo populacional em estudo, e os critérios de bout e epoch precisam ser relacionados ao constructo de AF em análise. Outra questão refere-se à utilização de análises baseadas em counts (dados proprietários específicos de cada aparelho) ou em dados brutos (aceleração bruta, expressa em equivalente gravitacional - " $g$ "). Enquanto os counts ainda possibilitam maior disponibilidade de evidências e comparabilidade com outros estudos, a segunda opção aumenta a transparência do processo analítico, permite uma maior comparabilidade dos dados entre diferentes marcas de acelerômetros e têm possibilitado o desenvolvimento de algoritmos de aprendizado de máquina (machine-learning) para classificação do tipo de AF (por exemplo, caminhada, atividades do dia-a-dia) ${ }^{1,4}$.

Um fator que tem suscitado debate é a utilização do diário de registro de uso. O diário é uma ferramenta auxiliar no processo de coleta e, inclusive, na análise dos dados, pois permite coletar informações como o período de utilização (datas e horários de colocação e retirada do aparelho) e de atividades específicas (por exemplo, período escolar, AF no tempo livre, aulas de Educação Física, tipo de AF). Tais informações permitem realizar recortes específicos nos dados identificando períodos do dia e, em alguns casos, a intensidade de AFs de interesse. Devido a complexidade analítica, a viabilidade da utilização de diários em estudos com amostras maiores é limitada.

Destacadas algumas das principais decisões que pesquisadores precisam tomar em seus estudos, cabe também ressaltar as limitações e os principais desafios da mensuração objetiva de AF e TS. Atividades aquáticas, ciclismo e qualquer atividade com sobrecarga (por exemplo, musculação, deslocamentos em aclives) não são captadas adequadamente pelos acelerômetros, podendo subestimar estimativas de $\mathrm{AF}^{2-3}$. Outra limitação é a ausência de informação específica do domínio no qual a AF foi realizada, o que impossibilita discriminar atividades domésticas, de trabalho, deslocamento ou lazer. Dessa maneira, estudos visando melhorar a mensuração dessas atividades são primordiais para futuras aplicações da acelerometria em estudos da área (por exemplo, algoritmos de reconhecimento de padrões de AF).

Com relação ao TS, a diferenciação da postura dos participantes (por exemplo, em pé, sentado ou deitado) é a principal limitação quando detectada ausência de movimento ${ }^{5}$. Além disso, o tempo de uso é um dos fatores que podem impactar as estimativas de TS. Presumivelmente, um maior tempo de uso resulta em estimativas mais fidedignas do TS, haja vista que possibilita caracterizar os dias de coleta 
de forma mais completa. Os algoritmos automatizados, como aqueles propostos por Choi et al. e Troiano et al., mostram robustez na eliminação de períodos de não uso do acelerômetro (por exemplo, 60 minutos consecutivos com ausência de aceleração) $)^{3}$. Apesar disso, os pesquisadores necessitam estabelecer critérios que possibilitem um equilíbrio entre perda de dados aceitável e nível de representatividade dos mesmos.

A última limitação que destacamos e, talvez, um dos maiores desafios atuais para os pesquisadores da área, é a comparabilidade frente a quantidade de decisões metodológicas necessárias e às diferentes marcas e modelos de acelerôme$\operatorname{tros}^{2-4}$. No cenário atual, a ampla variabilidade na redução de dados, oriunda de uma grande disponibilidade de pontos de corte, definição de total de dias e dias válidos, bem como outros procedimentos analíticos, desacelera o avanço esperado em nossa área. Evidentemente que a comparabilidade plena é utópica, mas muitas decisões metodológicas dentro da realidade financeira e logística de cada estudo poderiam ser mais bem padronizadas e cientificamente embasadas. Para isso é importante a elaboração de estudos metodológicos que evidenciem o impacto de "pequenas" tomadas de decisões nas estimativas finais.

Por fim, evidenciamos umas das consequências advindas da evolução e ampla utilização dos acelerômetros atualmente: a implementação do método sem a reflexão necessária. A definição sobre a utilização dos acelerômetros e as decisões metodológicas intrínsecas ao seu uso dependem fundamentalmente das perguntas de pesquisa e dos constructos de AF necessários para responde-las ${ }^{2,6}$. Os acelerômetros não necessariamente fornecem a melhor estimativa de AF para responder a todos os problemas de pesquisa. Algumas associações com desfechos de saúde, por exemplo, podem necessitar de informações mais específicas sobre o domínio no qual a AF foi realizada. Apesar dos potenciais vieses das medidas subjetivas, os questionários ainda podem ser a melhor alternativa para responder determinadas perguntas de pesquisa. Dessa forma, em estudos amplos que testam diferentes hipóteses, a associação de métodos objetivos e subjetivos tem sido identificada como a escolha mais adequada.

Reflexões semelhantes também são necessárias para as decisões metodológicas na utilização da acelerometria. Por exemplo, é necessário considerar a natureza intermitente da AF vigorosa em estudos sobre os efeitos da mesma na saúde de crianças, pois utilizar longos intervalos de epoch e bout subestimariam a prática de AF, bem como seu potencial efeito sobre a saúde. Outros grupos populacionais, como os idosos, e outros aspectos metodológicos como dias de mensuração, local de uso dos acelerômetros e utilização de diário, também dependem da pergunta de pesquisa como protagonista na tomada de decisão.

Todos esses pontos controversos e aspectos discutíveis sobre a utilização dos acelerômetros tornam o atual cenário desafiador, porém, profícuo! Observamos os pesquisadores brasileiros engajados em acompanhar as tendências internacionais na área. Dentre tais tendências, a demanda por dados objetivos de AF é sem dúvida uma das mais enfatizadas na atualidade. Os pesquisadores brasileiros têm respondido à altura e vêm buscando adquirir acelerômetros e aplica-los em seus estudos. É necessário utilizar esse "timing" para estabelecer um diálogo saudável entre os estudiosos da área, a fim de plantarmos uma semente para o futuro. Precisamos de maior robustez nas decisões metodológicas e maior comparabilidade entre os dados produzidos no Brasil. Por isso, além de maior padronização na redução de dados, é importante a condução de estudos multicêntricos que padronizem todo o processo de coleta de dados. É hora de ampliarmos a colaboração 
entre grupos de pesquisas brasileiros. Somente através de esforço conjunto conseguiremos obter um panorama mais fidedigno dos níveis de AF no país e os seus reais impactos na saúde pública.

\section{Referências}

1. Troiano RP, McClain JJ, Brychta RJ, Chen KY. Evolution of accelerometer methods for physical activity research. Br J Sports Med. 2014;48(13):1019-23.

2. Strath SJ, Kaminsky LA, Ainsworth BE, Ekelund U, Freedson PS, Gary RA, et al. Guide to the assessment of physical activity: Clinical and research applications: a scientific statement from the American Heart Association. Circulation. 2013;128(20):2259-79.

3. Pedisic Z, Bauman A. Accelerometer-based measures in physical activity surveillance: current practices and issues. Br J Sports Med. 2015;49(4):219-23.

4. Wijndaele K, Westgate K, Stephens SK, Blair SN, Bull FC, Chastin SF, et al. Utilization and Harmonization of Adult Accelerometry Data: Review and Expert Consensus. Med Sci Sports Exerc. 2015;47(10):2129-39.

5. Atkin AJ, Gorely T, Clemes SA, Yates T, Edwardson C, Brage S, et al. Methods of Measurement in epidemiology: sedentary Behaviour. Int J Epidemiol. 2012;41(5):1460-71.

6. Ainsworth B, Cahalin L, Buman M, Ross R. The current state of physical activity assessment tools. Prog Cardiovasc Dis. 2015;57(4):387-95. 